\title{
A suppressor of a centromere DNA mutation encodes a putative protein kinase (MCK1)
}

\author{
James H. Shero ${ }^{1}$ and Philip Hieter \\ Department of Molecular Biology and Genetics, Johns Hopkins University School of Medicine, Baltimore, \\ Maryland 21205 USA
}

\begin{abstract}
A new approach to identify genes involved in Saccharomyces cerevisiae kinetochore function is discussed. A genetic screen was designed to recover extragenic dosage suppressors of a CEN DNA mutation. This method identified two suppressors, designated MCK1 and CMS2. Increased dosage of MCK1 specifically suppressed two similar CEN DNA mutations in CDEIII, but not comparably defective CEN DNA mutations in CDEI or CDEII. A strain containing a null allele of $M C K 1$ was viable under standard growth conditions, had a cold-sensitive phenotype (conditional lethality at $11^{\circ} \mathrm{C}$ ), and grew slowly on Benomyl (a microtubule-destabilizing drug). Furthermore, when grown at $18^{\circ} \mathrm{C}$ or in the presence of Benomyl, the null mutant exhibited a dramatic increase in the rate of mitotic chromosome loss. The allele-specific suppression and chromosome instability phenotypes suggest that $M C K 1$ plays a role in mitotic chromosome segregation specific to CDEIII function. The $M C K 1$ gene encodes a putative protein-serine/threonine kinase, which suggests a possible role for the $M C K 1$ protein in regulating the activity of centromere-binding proteins by phosphorylation. MCK1 was identified and cloned independently for its involvement in the induction of meiosis and is identical to a gene that encodes a phosphotyrosyl protein with protein kinase activity.
\end{abstract}

[Key Words: S. cerevisiae; kinetochore function; CEN DNA; allele-specific suppression]

Received December 12, 1990; revised version accepted January 28, 1991.

The eukaryotic kinetochore is a complex macromolecular structure that plays a critical role in the segregation of chromosomes during mitosis and meiosis, providing the site of chromosome attachment to the spindle apparatus. Although the kinetochore is morphologically well defined in higher eukaryotes, little is known about specific proteins that are required for proper function. An understanding of how the structure mediates proper chromosome segregation will require the identification of gene products that are physically or temporally important for kinetochore function.

In Saccharomyces cerevisiae, single microtubules are seen directly attached to chromatin without structurally differentiated kinetochores (Peterson and Ris 1976). Nuclease digestion experiments of intact chromatin, however, demonstrate that the centromeric DNA exists in a highly resistant 250 -bp core that is flanked by nuclease hypersensitive sites (Bloom and Carbon 1982). It has been suggested that this core acts as the microtubule attachment site and may represent a primitive kinetochore structure. The centromere $(C E N)$ DNA from S. cerevisiae has been cloned (Clarke and Carbon 1980) and characterized extensively (for review, see Newlon 1988). Completely functional CEN DNA is only $\sim 120 \mathrm{bp}$ in length and contains three conserved centromere DNA

\footnotetext{
${ }^{1}$ Present address: Institute for Molecular Genetics, Baylor College of
} Medicine, Houston, Texas 77030 USA. elements (CDEs). An 8-bp conserved sequence (CDEI) is separated from a 25-bp conserved sequence (CDEIII) by a 78- to 86-bp AT-rich region (CDEII). Mutation and deletion analysis of several yeast centromeres indicates that CDEI and CDEII are not absolutely required but are important for optimal chromosome stability and that CDEIII is essential for centromere function. These studies indicate that understanding the role of gene products involved in CDEIII function will be critical for understanding kinetochore function. On this basis, investigators have begun biochemical characterization of proteins that specifically bind to CDEIII (Hegemann et al. 1986; $\mathrm{Ng}$ and Carbon 1987; Lechner and Carbon 1991). Here we describe a genetic strategy involving suppressor analysis to isolate genes important for $S$. cerevisiae kinetochore function. Our experiments were designed to identify genes that play a role in CDEIII function.

Suppressor analysis has proved to be useful for identifying protein-protein interactions in a variety of biological investigations (for review, see Botstein and Maurer 1982). In principle, if two proteins interact, a deleterious amino acid substitution in one protein can be suppressed by a compensatory change in the other. By starting with a mutation in a gene encoding one component of a system, interacting gene products can be identified. This method has been extended to DNA-protein interactions as well. For example, mutations in the SV40 origin of replication are suppressed by mutations in $\mathrm{T}$ antigen, a 
known origin-binding protein (Shortle et al. 1979). Another related strategy for identifying interacting proteins, termed dosage suppression, relies on overexpression of an extragenic wild-type gene to rescue a mutant phenotype (for examples, see Hayles et al. 1986; Hadwiger et al. 1989). It is reasonable to predict that dosage suppression may also be useful in identifying suppressors of mutations in binding sites for sequence-specific DNA-binding proteins. An underlying assumption in this strategy is that the DNA-binding site mutation causes a decrease in the binding affinity of the binding protein. Increasing the effective concentration of this protein would compensate for the lower binding affinity by mass action. The feasibility of this type of approach is supported by the experiments of Doto and Zinder (1984), which show that insertional mutations in the bacteriophage $\mathrm{fl}$ origin of replication are suppressed in trans by mutations that increase the concentration of an initiator protein.

Previously, we have analyzed a large set of CEN DNA mutations for their effects on the mitotic segregation of marker chromosome fragments, using a sensitive visual assay (Hegemann et al. 1988). A subset of these CEN mutations cause partial functional impairment, exhibiting a 10- to 50-fold increase in the rate of chromosome fragment loss. In this study, these partially defective CEN DNA mutant alleles were used as substrates in a dosage suppression screen. Two strains containing single base-pair insertions in CDEIII were transformed with a high-copy yeast genomic library and screened for transformants with improved function of the mutant centromere. These experiments identified two DNA sequences (CMS1 and CMS2, for centromere mutant suppressor) that act as extragenic dosage suppressors of specific CEN DNA mutations. These suppressors are excellent candidates for structural or regulatory components of the yeast kinetochore. In this discussion, we describe the characterization of the MCK1 gene (CMS1 was renamed $M C K 1$; see below), which encodes a protein with homology to known protein-serine/threonine kinases, and discuss a plausible model for its involvement in kinetochore regulation. In an independent investigation, Neigeborn and Mitchell (this issue) identified the same gene (initially named IME3) as a meiotic activator. The gene has therefore been renamed MCK1 (for centromere and meiosis regulatory kinase) to reflect our broader understanding of the roles that $M C K 1$ plays in controlling the fidelity of mitotic chromosome transmission and the induction of meiosis. Furthermore, the gene is identical to YPK1, a gene that encodes a $40-\mathrm{kD}$ phosphotyrosyl protein associated with protein tyrosine kinase activity (Dailey et al. 1990).

\section{Results}

Identification of dosage suppressors of CEN DNA mutations

Yeast strains YPH298 and YPH299 were used to identify sequences that at high copy number suppress the defect in chromosome segregation caused by single base pair insertions in CDEIII. These diploid strains contain mutant centromeres [YPH298, CDEIII(19ק20-T)]; YPH299, CDEIII(19ק20-G)] present on a SUP11-marked chromosome fragment for visual monitoring using a colony color assay (Shero et al. 1991). The effects of these mutations on chromosome fragment loss rates per cell division were determined quantitatively to be an increase of 10- and 20-fold above wild type, respectively. The frequency of red sectors in a pink colony is a qualitative measure of the frequency of mitotic chromosome fragment loss during the growth of the colony. Quantitative differences in chromosome fragment loss rates of as low as two- to threefold can be distinguished qualitatively by using this assay (Hegemann et al. 1988). We decided to use strains carrying CDEIII $C E N$ mutant alleles that retain partial centromere function for two reasons: (1) CDEIII has been shown by mutation and deletion analysis to be essential for centromere function; and (2) these mutations may be better candidates for suppression than mutations that abolish or drastically reduce centromere function.

The strains YPH298 and YPH299 were transformed with a library of total yeast genomic DNA cloned in a 2-micron/TRP1 high-copy plasmid vector (J.H. Shero, unpubl.). Approximately 10,000 (YPH299) and 12,000 (YPH298) $\mathrm{Trp}^{+}$transformant colonies were screened for a reduction in sectoring frequency. Two transformants of YPH299 and seven transformants of YPH298 displayed a reproducible suppressed red sectoring phenotype on replating. To determine whether the suppression phenotype was plasmid dependent, total DNA was prepared from each yeast transformant, plasmids were isolated by transformation of Escherichia coli, and each plasmid was then reintroduced into the yeast strain from which it was originally isolated. Only two plasmids were isolated that reproducibly displayed an increased-dosage suppression phenotype: pMCK1 (isolated from a YPH299 transformant) and pCMS2 (isolated from a YPH298 transformant). Restriction enzyme mapping and DNA hybridization demonstrated that inserts carried by these plasmids did not have DNA sequences in common (data not shown). $\mathrm{p} M C K 1$, which showed the strongest qualitative suppression phenotype (Fig. 1), was selected for detailed study.

MCK1 suppression phenotype is specific to two similar CEN DNA mutant alleles

To determine the specificity of the $M C K 1$ dosage suppression phenotype, the plasmids $\mathrm{p} M C K 1$ and vector alone were independently transformed into two strains containing trans-acting mutations that cause a high frequency of mitotic chromosome segregation errors (YPH628, ctf3/ctf3; YPH629, ctf9/ctf9; Spencer et al. 1990). The chromosome loss frequency was qualitatively monitored by replating two independent $\operatorname{Trp}^{+}$colonies on medium lacking tryptophan, allowing inspection of $\sim 100$ colonies for their sectoring phenotype. Estimates of chromosome fragment loss rates in the presence or 

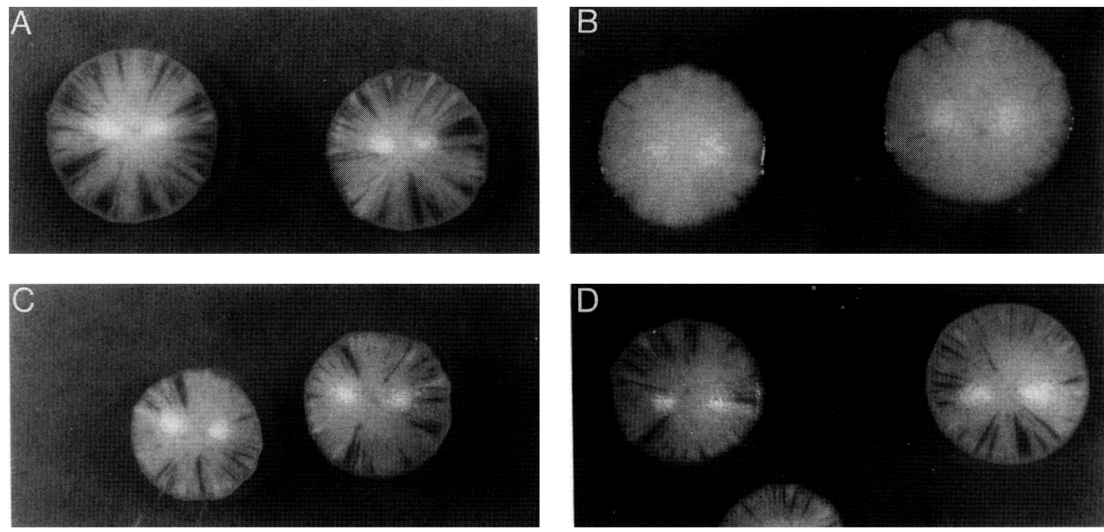

suppressed by pMCK1 is $5.9 \times 10^{-4}$. (C) Phenotype of a strain (YPH632) that contains a (CDEI $\Delta$ ) on the chromosome fragment and the plasmid vector pJS62 with no insert. $(D)$ Phenotype exhibited by YPH632 containing the $\mathrm{p} M C K 1$ extragenic dosage suppressor plasmid. Note the complete lack of a suppression phenotype with $\mathrm{p} M C K 1$ (cf. $C$ with $D$ ) in contrast to that seen with YPH299 (cf. $A$ with $B$ ).

absence of $\mathrm{p} M C K 1$ were confirmed quantitatively by fluctuation analysis (Table 1). These data showed that the chromosome loss phenotypes of YPH628 and YPH629 were not suppressed by pMCK1. This analysis confirmed that the suppression phenotype was due to a decrease in the rate of chromosome fragment loss per cell division and not due to an artifactual effect on the sectoring phenotypes. It also demonstrated that the suppression phenotype originally identified using YPH299 was not due to a global effect that suppressed all types of chromosome segregation defects.

$C E N$ mutant allele specificity of the MCK1 suppression phenotype was investigated by independently introducing vector alone (pJS62) and $\mathrm{p} M C K 1$ into 11 different

Figure 1. Colony phenotypes of strains carrying centromere DNA mutations in the presence or absence of $\mathrm{p} M C K 1$ suppressor. (A) Phenotype of the parent yeast strain (YPH299) that contains the 2-micron/TRP1 high-copy plasmid vector (pJS62) with no DNA insert. This strain is homozygous for the ade2-101 mutation and contains a URA3/SUP11-marked chromosome fragment with a $C E N$ DNA mutation [CDEIII $(19 \nabla 20-G)]$ that is lost at a rate of $3.8 \times 10^{-3}$ per cell division giving rise to red sectors. $(B)$ Phenotype exhibited by the YPH299 strain containing the $\mathrm{p} M C K 1 \mathrm{ex}-$ tragenic dosage suppressor plasmid. The chromosome fragment loss rate for YPH299 Phenotype exhibited by YPH 632 containing
on phenotype with $\mathrm{p} M C K 1$ (cf. $C$ with $D$ ) in 


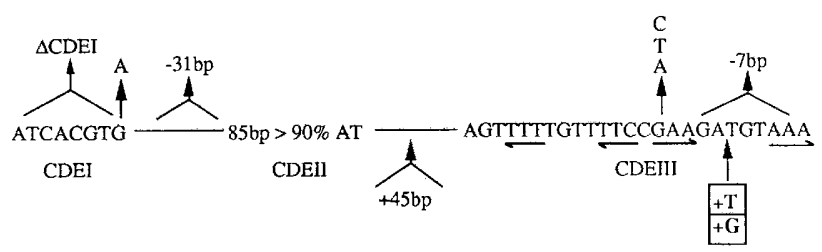

Figure 2. Summary of CEN DNA mutant allele specificity for suppression by $\mathrm{p} M C K 1$. The conserved regions /CDEI, CDEII, CDEIII) of the CEN6 DNA are indicated. CEN mutant alleles, analyzed for suppression by $\mathrm{p} M C K 1$, are shown above and below the CEN6 sequence. Arrows pointing away from the CEN6 sequence indicate DNA base-pair changes (with the change shown) or deletions (indicated by $\Delta$ ). Arrows pointing toward the DNA sequence indicate insertion mutations. Mutant alleles suppressed by $\mathrm{p} M C K 1$ are boxed; all other alleles tested were not significantly suppressed by $\mathrm{p} M C K 1$.

$3.8 \times 10^{-3}$ compared with $5.9 \times 10^{-4}$ when suppressed by $\mathrm{p} M C K 1$. This sixfold quantitative effect is clearly distinguishable qualitatively in sectoring colonies using the color assay (Fig. 1A,B). Similarly, the chromosome loss phenotype caused by the CDEIII $(19 \nabla 20-T)$ was suppressed fivefold by the high-copy $\mathrm{p} M C K 1$. Four additional CDEIII mutants causing either drastic $(500-$ to 1000 -fold/ increases in chromosome fragment loss rates or complete loss of $C E N$ function were not suppressed, as judged by the qualitative assay. It is possible that $\mathrm{p} M C K 1$ is unable to compensate for CDEIII mutations that cause an extreme reduction in the affinity of a DNA-binding protein for its binding site. Perhaps the most informative $C E N$ mutant alleles tested for suppression by $\mathrm{p} M C K 1$ were those in CDEI and CDEII. The segregation defects associated with these $C E N$ mutant alleles are all comparable in magnitude to the original CDEIII mutation used in isolating $M C K 1$. Introduction of the high-copy $\mathrm{p} M C K 1$ caused little or no qualitative (Fig. 1C, D) or quantitative (Table 1) suppression of chromosome fragment loss rates in four strains that carried either a CDEI mutation (two alleles tested) or a CDEII mutation (two alleles tested), although the mutants caused only moderate increases in chromosome loss ( $\sim 5$ - to 25 -fold). These data suggest that MCK1 plays a role in chromosome segregation that is specific to CDEIII function.

\section{Molecular characterization of MCK1}

The suppressor activity of $\mathrm{p} M C K 1$ was carried on a $7-\mathrm{kb}$ fragment derived from a partial Sau3A restriction digest of yeast genomic DNA and was localized to a $3.6-\mathrm{kb}$ BamHI-ClaI fragment. Unique restriction sites were modified to create frameshift mutations, and plasmids were assayed for suppressor activity by transforming into YPH299, followed by qualitative scoring of the sectoring frequency. A frameshift mutation introduced at the $\mathrm{NcOI}$ restriction site (underlined in Fig. 4, below) destroyed suppressor activity of the plasmid, indicating that the NcoI site was likely to be located in the amino acidcoding sequence of the MCK1 gene.

\section{Physical and genetic mapping of MCK1}

A radioactive probe was made from the cloned MCK1 gene and hybridized to a Southern blot containing yeast chromosome-sized DNA molecules. This provided unambiguous assignment of $M C K 1$ to chromosome XIV (data not shown). MCK1 was physically positioned on chromosome XIV by the chromosome fragmentation method (Vollrath et al. 1988; Gerring et al. 1990) and by probing the resultant chromosome fragments with probes proximal and distal to the site of fragmentation. This placed $M C K 1 \sim 80 \mathrm{~kb}$ from the left arm telomere of chromosome XIV (Fig. 3). Standard meiotic mapping methods placed the $M C K 1$ gene $18 \mathrm{cM}$ proximal to the pha2 locus and indicated that the MCK1 gene had not been identified previously.

MCK1 deduced amino acid sequence is homologous to protein kinases

The DNA sequence of a 1.7-kb region included the entire $M C K 1$ open reading frame. This open reading frame is encoded by $1125 \mathrm{bp}$ and predicts a protein of 375 amino

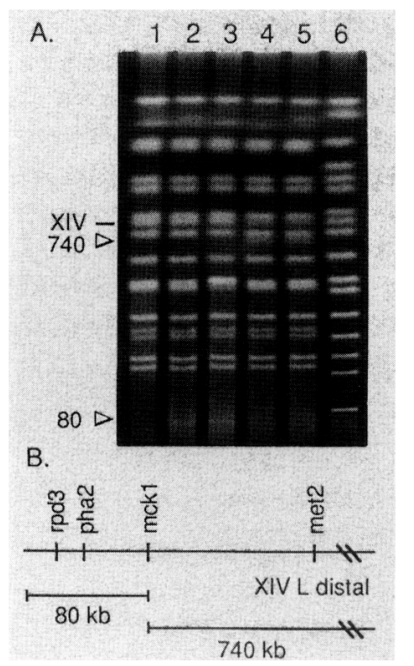

Figure 3. Physical and genetic mapping of $M C K I$. The cloned MCK1 DNA segment was used to fragment chromosome XIV into chromosome fragments containing sequences proximal or distal to the cloned segment. This placed the MCK1 locus $\sim 80$ $\mathrm{kb}$ from the telomere of its own chromosome arm and $\sim 740 \mathrm{~kb}$ from the telomere on the opposite chromosome arm. Assignment to the left arm was accomplished by probing Southern blots of chromosome fragment containing strains with the $K A R 1$ gene (which hybridized to the $740-\mathrm{kb}$ fragment) and the $R P D 3$ gene (which hybridized to the $80-\mathrm{kb}$ fragment). (A) Electrophoretic karyotypes of strains carrying chromosome fragments. Chromosome XIV is indicated by a dash; chromosome fragments are indicated by open arrowheads. (Lane 1) Parental strain; (lanes 2 and 3) strains carrying MCK1-distal chromosome fragments $(80 \mathrm{~kb})$; (lanes 4 and 5) strains carrying $M C K 1-$ proximal chromosome fragments (740 kb); (lane 6) YPH149 as size standard. $|B|$ Diagram of extreme distal end of the chromosome XIV left arm. MCK1 was placed $18 \mathrm{cM}$ proximal to pha2 by standard tetrad analysis. 
acids with a $M_{\mathrm{r}}$ of 43,108 (Fig. 4). Computer searches using the program FASTA (Lipman and Pearson 1985) to look for amino acid sequences similar to $M C K 1$ in standard data bases /GenBank release 57.0 and NBRF/PIR release 18.0) revealed significant homology to numerous protein kinases. Typical homology scores obtained showed $\sim 25 \%$ identity between the predicted $M C K 1$ protein and many known protein kinases in the data bank (e.g., $C D C 28$ ). Outside of the 11 defined protein kinase motifs (for review, see Hanks et al. 1988), however, there was no significant sequence similarity. A personal data base maintained by Mark Goebl at Indiana University showed significantly higher homologies to two recently published sequences. Glycogen synthase kinase-3 from rat (GSK-3; Woodgett 1990) and shaggy from Drosophila melanogaster (sgg, Bourouis et al. 1990) are $40 \%$ and $38 \%$ identical, respectively, over the length

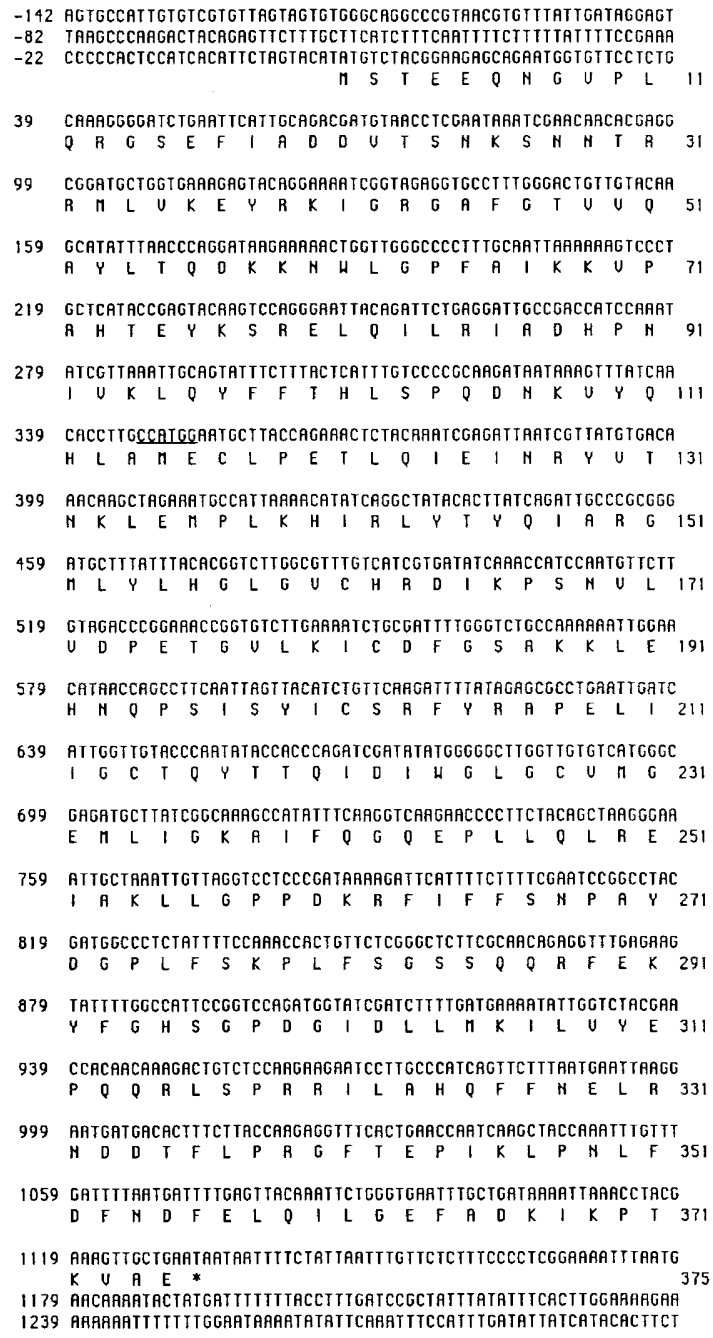

Figure 4. Nucleotide sequence of the $M C K 1$ gene and flanking DNA. The predicted amino acid sequence is shown in singleletter code with a star representing the termination codon. The Ncol restriction enzyme cleavage site used to generate mck1::HIS3 is underlined. of $M C K 1$ (Fig. 5A). They are $45 \%$ (GSK3) and $41 \%$ (sgg) identical in the catalytic domain (shown as the amino acids between number signs (\#) in Fig. 5A). This sequence analysis predicts that $M C K 1$ encodes a protein that is likely to be a protein-serine/threonine kinase.

In addition, an unusual sequence similiarity was identified between MCK1 and TPK1. TPK1 (Toda et al. 1987) is a yeast protein kinase with a characteristic homology to $M C K 1(\sim 25 \%)$ in the catalytic domain. The rest of the gene shows almost no homology to MCK1, with the striking exception of the first 8 amino acids, which are identical (C. Chan, pers. comm.). A computer search ( $M$. Goebl, pers. comm.) did not reveal any additional protein sequences with a similiar 8-amino-acid motif. Although the function of this sequence is unknown, its aminoterminal position suggests that it may be a signal sequence or a site for protein modification.

\section{Gene disruption of MCK1}

Because a frameshift mutation at the $N c o$ I restriction site completely abolished suppression, a DNA fragment containing the HIS3 gene was cloned into this site to create an insertion mutant allele. Sequence analysis showed that this allele would be expected to result in a null mutation owing to disruption of the catalytic domain. A diploid strain that contained a SUP11-marked chromosome fragment (YPH273) was transformed with a linear DNA fragment containing the mck1::HIS3 allele. Two independently derived strains, in which one copy of $M C K 1$ was replaced with $m c k 1:: H I S 3$, were sporulated and dissected. Analysis of resultant tetrads showed that the majority contained four viable spores with the HIS3 disruption marker segregating $2+: 2-$. Furthermore, spore colonies containing mck1::HIS3 had normal growth rates and did not exhibit a qualitative increase in the loss rate of the chromosome fragment. Southern hybridization analysis of genomic DNA from the parent diploid and four spore colonies of a tetrad confirmed correct disruption of $M C K 1$ at the chromosomal locus /data not shown). Low-stringency Southern hybridization experiments using the $3.6-\mathrm{kb}$ BamHI-ClaI genomic fragment as a probe indicated that a single copy of $M C K 1$ was present in the haploid yeast genome (data not shown). The MCK1 gene product is therefore not required for viability or proper chromosome segregation under standard laboratory growth conditions $\left(30^{\circ} \mathrm{C}\right)$.

\section{Phenotypes associated with MCK1 gene disruption allele}

The mck $1:: H I S 3$ disruption strain was examined for phenotypes that might provide additional information toward understanding the function of the MCK1 gene product. Two strains carrying the mck1::HIS3 allele (YPH635, containing a SUP11-marked chromosome fragment, and YPH636) were tested for conditional growth at various temperatures and found to be completely inviable at $11^{\circ} \mathrm{C}$. To test for potential chromosome segrega- 
Figure 5. The $M C K 1$ gene encodes a putative protein kinase. (A) Alignment of MCK1 with glycogen synthase kinase-3 (GSK-3 from rat; Woodgett 1990) and shaggy (sgg, from D. melanogaster; Bourouis et al. 1990) are shown. MCK1 is $45 \%$ (GSK3) and $41 \%$ (sgg) identical to these proteins over the canonical protein kinase catalytic domain (shown as the amino acids between number signs (\#). The conserved and invariant residues identified by Hanks et al. (1988) are highlighted by dots above the appropriate residues. MCK1 encodes a novel protein that is likely to be a protein-serine/threonine kinase. (B) An unusual sequence identity was identified between $M C K 1$ and TPKI. The first 8 amino acids of these two genes are identical yet they show no homology outside of the catalytic domain in the remainder of the sequence. Although the function of this sequence is unknown, its amino-terminal position suggests that it may be a signal sequence or a site for protein modification.

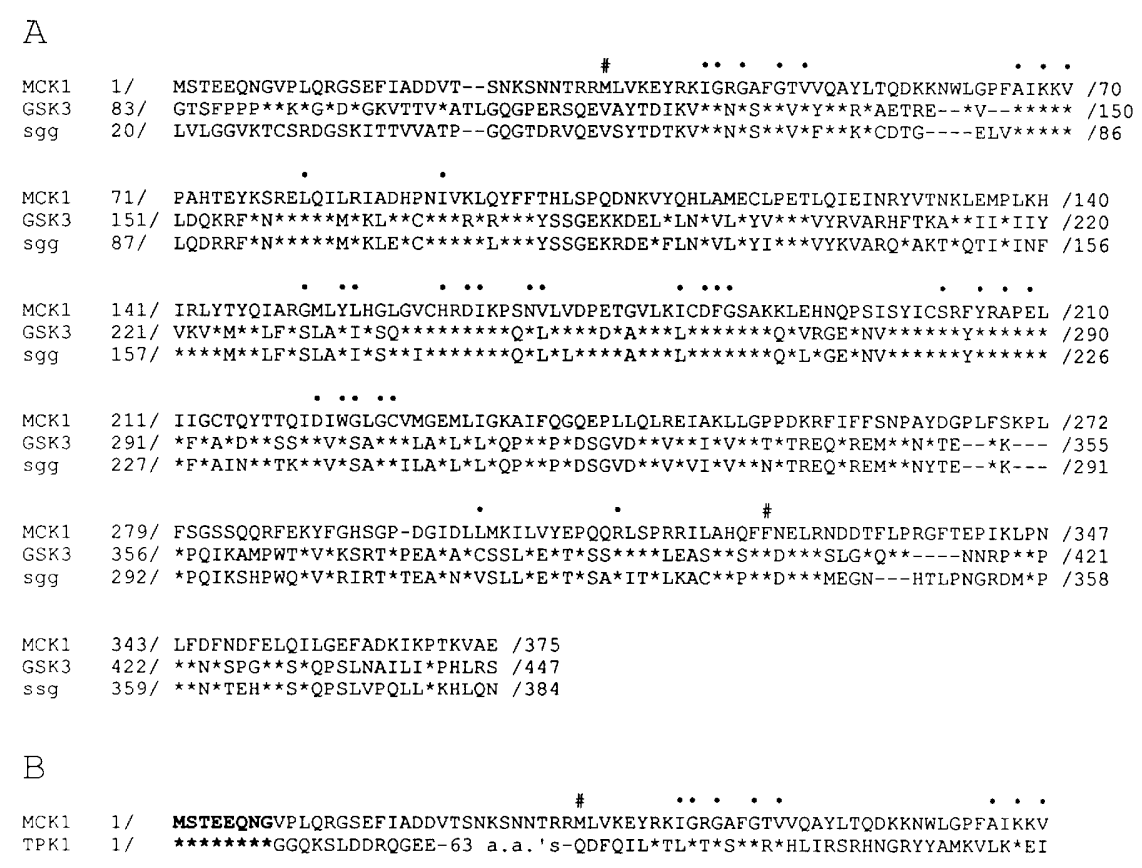

A

MCK1 1/ MSTEEQNGVPLQRGSEF IADDVT--SNKSNNTRRMLVKEYRKIGRGAFGTVVQAYLTQDKKNWLGPFFAIKKV /70

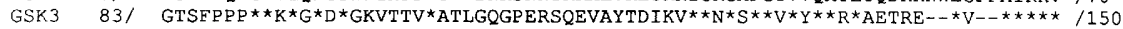

MCK1 $71 /$ PAHTEYKSRELQILRIADHPNIVKLQYFFTHLSPODNKVYOHLAMECLPETLQIEINRYVTNKLEMPLKH $/ 140$

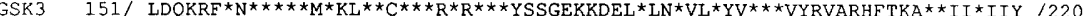

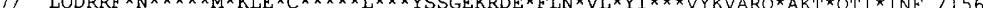

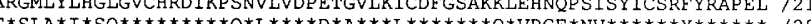

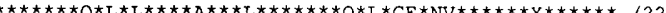

11/ IIGCTQYTTQIDIWGLGCVMGEMLIGKAIFQGQEPLLQLREIAKLLGPPDKRF IFFSNPAYDGPLFSKPL $/ 272$

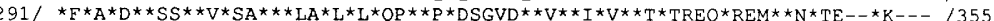

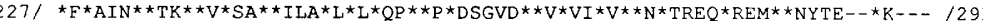

279/ FSGSSQQRFEKYFGHSGP-DGIDLLMKILVYEPQQRLSPRRILAHQFFNELRNDDTFLPRGFTEPIKLPN /347

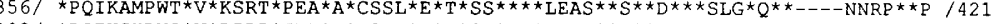

343/ LFDFNDFELQILGEFADKIKPTKVAE /375

4227 $\star \star N^{\star} \mathrm{SPG} \star \star S^{\star} \mathrm{QPSLNAILI} \star \mathrm{PHLRS} / 447$

$\star \star \star N * T E H \star \star S^{\star} Q P S L V P Q L L \star K H L Q N / 38$

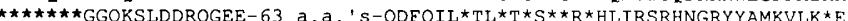

tion defects at a semipermissive temperature, YPH635 (mck1::HIS3) and YPH637 (MCK1 wild-type control) were plated to single cells at $14^{\circ} \mathrm{C}, 16^{\circ} \mathrm{C}$, and $18^{\circ} \mathrm{C}$. YPH635 formed colonies at these temperatures but showed an increase in chromosome fragment loss rate when compared to YPH637 (Fig. 6).

Benomyl is a microtubule-destabilizing drug that has been used recently in a genetic screen to identify genes important for microtubule function (Hoyt et al. 1990, Stearns et al. 1990). These investigators isolated and characterized three genes that mutate to alleles exhibiting supersensitivity to Benomyl at normally sublethal concentrations. Because $\beta$-tubulin is believed to be the only intracellular target of Benomyl, these genes are considered excellent candidates for participants in a pathway involving microtubules. Therefore, we tested mck1 strains for sensitivity to Benomyl. This analysis revealed that mck1 strains showed reduced growth on Benomyl-
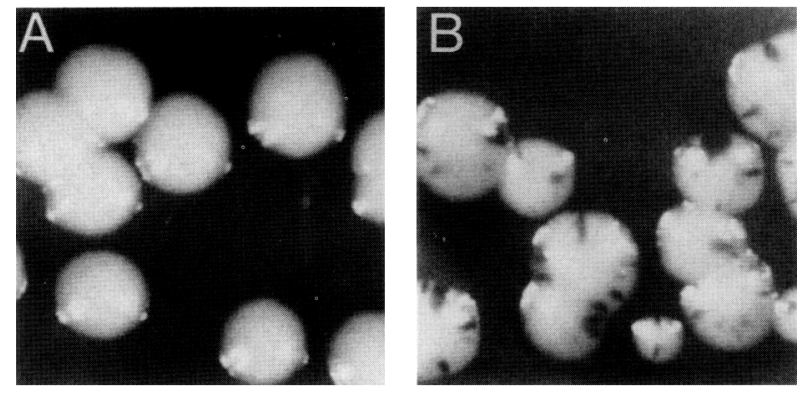

Figure 6. Sectoring phenotypes exhibited by haploid strains containing either $M C K 1$ or $M C K 1:: H I S 3$ at $16^{\circ} \mathrm{C}$. (A) $M C K 1$ (wild type); (B) mck1::HIS3 (mutant). containing medium (Fig. 7). Furthermore, when a mck1::HIS3 strain (YPH635) containing a SUP11marked chromosome was sparsely dispersed on plates that contained Benomyl and incubated at $25^{\circ} \mathrm{C}$, a dramatic increase in chromosome fragment loss was observed in the resultant colonies as compared with the appropriate wild-type strain (YPH637).

\section{Discussion}

We have described an experimental approach to isolate trans-acting factors that are required for proper kinetochore function in $S$. cerevisiae. Our strategy relied on a series of strains containing CEN DNA mutations that were characterized for their effects on mitotic chromosome fragment stability in vivo using a colony color assay. Two yeast strains containing partially defective centromeres (CDEIII mutations) were used to identify transacting suppressors of the centromere defect. We used a dosage suppression screen, reasoning that overexpression of a CEN DNA-binding protein, or its positive regulator(s), may suppress the CEN DNA mutation. A highcopy yeast genomic library (constructed in a $2 \mu$ vector) was used to transform these strains, which were then screened visually for improvement in mutant $C E N$ function. Two DNA sequences (MCK1 and CMS2) were identified as extragenic dosage suppressors and are excellent candidates for structural or regulatory components of the yeast kinetochore.

Allele specificity of suppression is a useful criterion to distinguish interaction suppressors (generally allele specific) from bypass suppressors (generally allele nonspecific). Increased $M C K 1$ dosage suppressed two similar CDEIII mutations but failed to suppress eight other CEN 


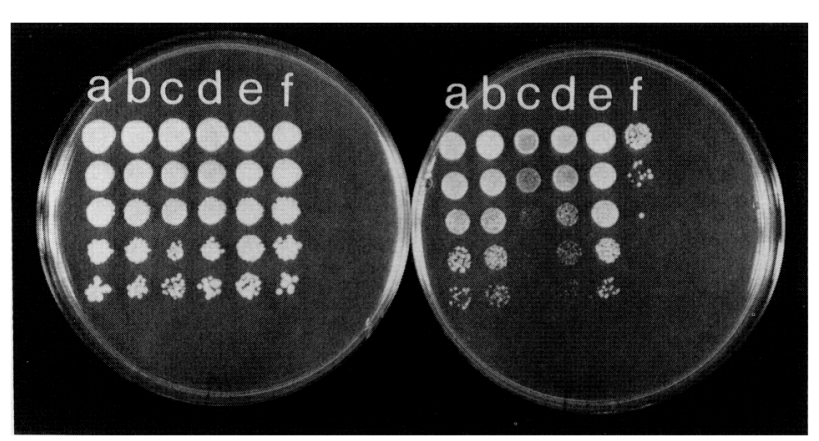

Figure 7. Serial dilution patch test for Benomyl sensitivity. The two plates shown have been inoculated with the same ordered serial dilution grid of yeast cells (shown from top to bottom). (Right) Plate containing $10 \mu \mathrm{g} / \mathrm{ml}$ of Benomyl (a drug concentration that does not significantly affect the growth rate of the wild-type parent strains); (left plate serving as a control. Both plates were incubated for 3 days at $25^{\circ} \mathrm{C}$. In rows $a-d$, the four haploid products generated by tetrad dissection of a diploid strain (YPH633) heterozygous for the MCK1 disruption are analyzed. Lanes $a$ (YPH634) and $b$ (YPH637) contain the wildtype MCK1 locus; lanes $c$ (YPH635) and d (YPH636) contain the mck1::HIS3 disruption; lane $e$ contains a MCK1 wildtype strain (YPH278); lane $f$ contains a tub3::TRP1 strain (DBY2288) that is known to be Benomyl sensitive. This analysis clearly shows that the mck $1:$ HIS3 disruption causes a slowgrowth phenotype on Benomyl.

DNA mutations. In particular, mutations in CDEI and CDEII that cause a segregation defect of comparable magnitude to the suppressible CDEIII mutant were not suppressed by $\mathrm{p} M C K 1$. We interpret this allele specificity to mean that suppression by the $M C K 1$ protein kinase acts in a pathway important for CDEIII function rather than in a more global pathway that would suppress segregation defects in general.

Replacement of the wild-type MCK1 gene by mck1:: HIS3 demonstrated that it is not essential for growth at $30^{\circ} \mathrm{C}$. The $M C K 1$ insertion mutant exhibited two potentially informative secondary phenotypes: conditional lethality at $11^{\circ} \mathrm{C}$ and slow growth on Benomyl. Furthermore, when grown at semipermissive temperatures, or in the presence of Benomyl, the MCK1 insertion mutant exhibited an increase in the loss rate of a wildtype $C E N$-containing chromosome fragment. These data indicated that the endogenous copy of MCK1 functions in a biochemical pathway that is important for mitotic chromosome segregation. Because the only known intracellular target of Benomyl is $\beta$-tubulin (Thomas et al. 1985), the finding that mck1:: HIS3 is hypersensitive to this drug implies that it is important for microtubule function. MCK1 may be important for spindle microtubule stability, for example, by phosphorylating a microtubule-associated protein. In this model, suppression of the CDEIIII centromere mutation (which itself may decrease the stability of interactions with microtubules) would be explained by a compensatory increase in the overall stability of microtubules. Consistent with this notion is the fact that microtubules are destabilized in the cold and the observation that the mck1-disruption strain is cold sensitive.

DNA sequence analysis reveals that the $M C K 1$ gene encodes a putative protein-serine/threonine kinase by virtue of its homology to known protein kinases (see also Dailey et al. 1990; Neigeborn and Mitchell, this issue). We anticipated that the dosage suppression screen might identify genes that code for CDEIII DNA-binding protein(s) or positive regulators of these binding proteins. The fact that the MCK1 gene product has been shown to have protein kinase activity (Dailey et al. 1990) makes the latter suggestion particularly attractive. There are several examples in the published literature of DNAbinding proteins that are activated upon phosphorylation (Magasanik 1988; Prywes et al. 1988; Yamamoto et al. 1988; Raychauduri et al. 1989|. Furthermore, numerous studies provide evidence for changing protein phosphorylation states in premitotic and mitotic cells. There are several proteins that are phosphorylated specifically at the late $G_{2}$ stage of the cell cycle and then rapidly dephosphorylated shortly after mitosis (Westwood et al. 1985; Lohka et al. 1987; for review, see Nurse 1990). Of particular interest in the present study is the finding that microtubule-organizing centers (including kinetochores) contain phosphoproteins (Davis et al. 1983; Vandre et al. 1984). The regulation of mitotic events by phosphorylation also implies that dephosphorylation of the same residues will have an opposing regulatory contribution. Recently, Axton et. al. (1990) demonstrated that one of the protein phosphatase 1 isoenzymes in Drosophila is essential for mitosis and that mutations in this gene cause defective spindle organization, abnormal sister chromatid segregation, hyperploidy, and excessive chromosome condensation.

Importantly, Lechner and Carbon (1991) have obtained biochemical evidence in $S$. cerevisiae that at least one protein(s) within a bound complex on CDEIII is a phosphoprotein. They have shown that phosphorylation of this protein(s) is necessary for complex formation. It is reasonable to propose that the intracellular target of the MCK1 kinase may be the same phosphorylated protein species that Lechner and Carbon have identified. This implies a role for the $M C K 1$ kinase in converting an inactive CEN DNA-binding protein into an active form. Alternatively, MCK1 kinase could act in a multistep pathway (e.g., as a regulator of a protein upstream of the active CEN DNA-binding protein). The latter notion is perhaps easier to reconcile with the work of Neigeborn and Mitchell (this issue), which assigns at least two additional functions of the MCK1 kinase: transcriptional activation of $I M E 1$ and the stimulation of spore maturation.

MCK1 kinase appears to play a positive regulatory role in both mitotic centromere function and activation of early meiotic gene expression. It is possible that MCK1 kinase modulates the activity of a variety of intracellular target proteins that operate in multiple unrelated biochemical pathways. Alternatively, MCK1 kinase may regulate a protein (or proteins) common to both the centromere and the meiosis pathways. A precedent exists 
for a DNA sequence-specific binding protein, called CBF1 or CP1, which plays an important functional role for both centromere activity and transcription (Bram and Kornberg 1987; Baker et al. 1989; Jiang and Philippsen 1989; Cai and Davis 1990). CBF1 (CP1) protein binds to CEN DNA at CDEI and also interacts at sites upstream of genes required for methionine prototrophy. By analogy, MCK1 may regulate the activity of a protein that is a CDEIII DNA sequence-specific binding protein (functioning within the kinetochore complex) and that may also play a role in regulation of transcription in meiotic cells (through interactions with other components). Finally, there may be an obligatory relationship between the proper assembly of a meiotic centromere complex (early after commitment to meiosis) and subsequent progression through the meiosis program. In this scenario, the defective assembly or function of kinetochore proteins at the centromere might cause a meiotic "delay" at an early step after entry into meiosis. This delay might be observed as the altered transcriptional activation of genes normally expressed early and subsequent defects in ascus formation. The delay could be due to a structural defect or to a surveillance system that monitors the successful completion of steps before progression to others. Such a "checkpoint" control mechanism has been described for cell cycle progression in $G_{2}$ of mitotic cells in response to radiation-induced DNA damage (Hartwell and Weinert 1989).

Our experiments were inspired by the finding that mutations in the SV40 origin of DNA replication are suppressible by missense mutations in the origin-binding protein (Shortle et al. 1979). The small genome size of SV40 and the cold-sensitive phenotype of the origin point mutations facilitated this effort, allowing efficient mutagenesis of the $\mathrm{T}$ antigen gene and direct selection of second-site revertants. Here we show that this type of approach may also identify unknown components of a pathway by the isolation of CEN DNA mutation suppressors from $S$. cerevisiae. Using a dosage suppression strategy, the isolation of suppressors from a genome the size of yeast is feasible without requiring a selection procedure. Demonstration that MCK1 encodes a protein involved in proper chromosome segregation validates this approach. A similar strategy (provided a suitable assay is available) may be useful in identifying trans-acting factors important for other DNA protein interactions as well.

\section{Materials and methods}

Strains and methods

The $E$. coli strain DH5 $\alpha$ (Bethesda Research Laboratories) was used for routine cloning and library construction. Standard recombinant DNA techniques were carried out as described (Maniatis et al. 1982). Transformations were performed by the method of Hanahan (1983). Miniprep DNA samples were isolated for analysis by the boiling method of Holmes and Quigley (1981). Table 2 lists the genotypes of all yeast strains used in this work. YPH strains are isogenic derivatives of YNN217 (original source, M. Carlson), an S288C derivative. Additional auxotrophic markers have been introduced by one-step gene replacement as described (Sikorski and Hieter 1989). Benomylsensitive strains were obtained from sources shown. Standard media recipes were from Sherman et al. (1978). Minimal media plates were supplemented with auxotrophic requirements, as described (Sherman et al. 1978), with a limiting adenine modification (Hieter et al. 1985) for colony color indicator plates. Yeast transformations were done by the lithium acetate procedure (Ito et al. 1983), with minor modifications. Transformants were selected on synthetic complete medium (Sherman et al. 1978) lacking either histidine, or tryptophan, depending on the gene under selection. Benomyl (gift from DuPont) sensitivity was assayed qualitatively by patch tests as ordered serial dilutions containing from $10^{5}$ to $10^{1}$ cells per patch (as described by Spencer et al. 1990) on YPD plates containing $10 \mu \mathrm{g} / \mathrm{ml}$ of Benomyl (prepared as described; Stearns et al. 1990). Genetic analysis was performed by using standard protocols /Sherman et al. 1978). Yeast genomic DNA was isolated for restriction digest and plasmid rescue into $E$. coli, as described (Davis et al. 1980).

Measurement of chromosome fragment loss rates by visual inspection and fluctuation analysis

Use of a colony color assay (Hieter et al. 1985) for qualitative measurement of chromosome fragment loss rates by visual inspection of colony-sectoring frequency was described in detail previously (Hegemann et al. 1988). The ability to distinguish quantitative differences in loss rates of as little as two- to threefold by visual inspection of colony sectoring phenotype has been thoroughly documented.

Fluctuation analysis by the method of the median (Lea and Coulson 1949) was performed as described (Hegemann et al. 1988), with the following modifications: A preincubation of logarithmically growing cells in $1 \mu \mathrm{g} / \mathrm{ml}$ of 5 -fluoro-orotic acid $\left(5^{\prime}\right.$-FOA $)$ in sterile distilled $\mathrm{H}_{2} \mathrm{O}\left(4 \mathrm{hr}\right.$ at $4^{\circ} \mathrm{C}$ with gentle shaking/ eliminated the leaky phenotype of this negative selection, which was reported previously (Hegemann et al. 1988). Therefore, we used the URA3 marker contained on the chromosome fragment to determine mitotic loss rates. Colonies were obtained for fluctuation, as described (Hegemann et al. 1988), and were placed in $200 \mu \mathrm{l}$ of $\mathrm{H}_{2} \mathrm{O}$ containing $\mathrm{l} \mu \mathrm{g} / \mathrm{ml}$ of $5^{\prime}$-FOA and disrupted to single cells by vortexing. A $20-\mu l$ aliquot was removed immediately, and a 100 -fold serial dilution $(100 \mu l)$ was plated to indicator plates to determine the number of cells per colony. The remainder of the colony was incubated for $6 \mathrm{hr}$ at $4^{\circ} \mathrm{C}$ with gentle shaking, and $100 \mu \mathrm{l}$ (representing $50 \%$ of the original colony) was plated to supplemented minimal plates with $1 \mu \mathrm{g} / \mathrm{ml}$ of $5^{\prime}$-FOA (Boeke et al. 1987) to determine the number of cells that had lost the URA3/SUP11-marked chromosome during colony growth. Calculation of the mitotic chromosome fragment loss rates found in Table 1 was done by the method of the median (Lea and Coulson 1949).

\section{Genetic screen for CEN DNA mutant suppressors}

The strains used for suppression analysis were YPH298 and YPH299 (for genotypes, see Table 3), which show a 10- and 20 -fold increase, respectively, in chromosome fragment loss rates. To isolate yeast genomic sequences that suppress the effect of the CEN mutations, a yeast genomic library was constructed in a TRP1/2-micron shuttle vector, pJS62 (J.H. Shero, unpubl.). The yeast strains were transformed with $6 \mu \mathrm{g}$ of library DNA by the LiAc procedure (Ito et al. 1983), diluted, and plated onto supplemented minimal medium lacking tryptophan at a density yielding $\sim 200$ transformants per plate. Transformed cells were allowed to form colonies at $30^{\circ} \mathrm{C}$ for 5 days and then 
Table 2. List of yeast strains for this study

\begin{tabular}{|c|c|c|}
\hline Strain & Genotype & Source \\
\hline YPH273 & 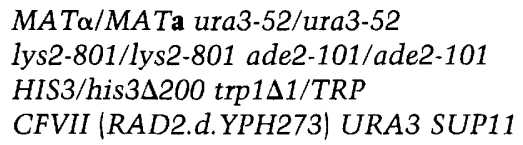 & this study \\
\hline YPH278 & 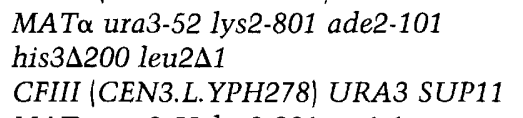 & Spencer et al. (1990) \\
\hline DBY2288 & $\begin{array}{l}\text { MAT } \alpha \text { ura3-52 lys2-801 trp1-1am } \\
\text { his } 3 \Delta 200 \text { leu2-3,112 tub3::TRP1 }\end{array}$ & Botstein laboratory \\
\hline YPH634 & $\begin{array}{l}\text { MATa ura3-52 lys2-801 ade2-101 } \\
\text { his3 } 3200 \operatorname{trp} 1 \Delta 1 \text { leu2 } 1\end{array}$ & this study \\
\hline YPH635 & 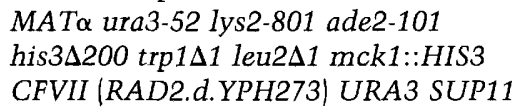 & this study \\
\hline YPH636 & 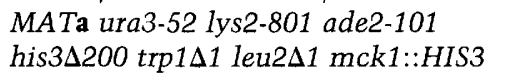 & this study \\
\hline YPH637 & 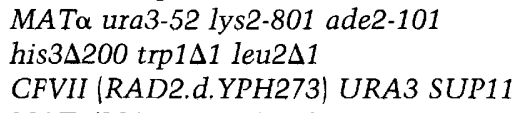 & this study \\
\hline YPH633 & 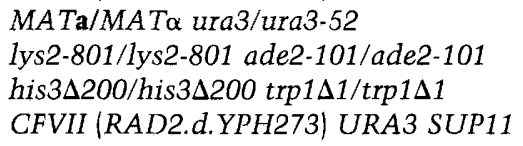 & this study \\
\hline YPH628 & 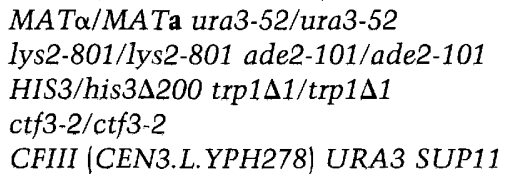 & Spencer et al. (1990) \\
\hline YPH629 & 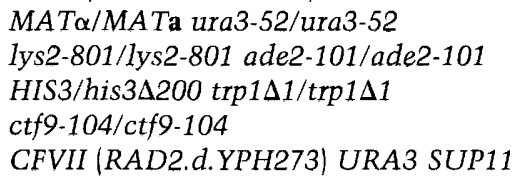 & Spencer et al. $(1990)$ \\
\hline
\end{tabular}

transferred to $4^{\circ} \mathrm{C}$ for $\sim 10$ days to allow optimal color development. The colonies were then screened with the aid of a dissecting microscope (Wild M5A M5 APO, Wild Heerbrugg, Switzerland) for a reduction in sectoring frequency that may have indicated mitotic stabilization of the chromosome fragment. For YPH298, 12,000 transformants were screened to obtain 23 putative suppressors; and for YPH299, 10,000 transformants were screened to obtain 9 putative suppressors. These transformants were streaked to fresh minimal medium lacking tryptophan to obtain colony-pure strains, serially diluted, replated to a density of 50 cells per plate, and incubated (as above) to allow colony color development. By inspecting an entire field of colonies on a plate for a reduction in sectoring frequency compared with the same strain transformed with vector (pJS62) alone we identified nine true positives (seven for YPH298 and two for YPH299| and discarded the others as false positives. Plasmids were rescued from these strains into $\mathrm{DH} 5 \alpha$ (Davis et al. 1980) and transformed back into the strains they were isolated from. By these criteria only two plasmids were recovered that demonstrated a reproducible suppression phenotype. The plasmids were named pMCK1 (formerly pCMS1), originally isolated from YPH299, and pCMS2, originally isolated from YPH298.

Mutant allele (cis or trans) specificity was determined for $M C K 1$ by transforming vector alone (pIS62) and pMCK1 into 2 strains containing homozygous trans-acting mutations, 10 strains containing cis-acting (CEN DNA) mutations, and 1 strain containing a wild-type centromere (strain genotypes listed in Table 3). Two independent transformants for each were visually assayed for suppressor activity (as above). In addition, three CEN DNA mutations that completely abolished centromere function were tested for suppression by $\mathrm{p} M C K 1$ as follows: Red segregant strains (red color reflects loss of the URA3/ SUP11/CEN6 chromosome fragment) were picked from YPH299 that contained either pIS62 or pMCK1. These strains were transformed with chromosome fragmentation vectors pJS13, pJS14, and pIS15, as described (Hegemann et al. 1988), and four independent transformants were visually assayed for the restoration of $C E N$ function (as above).

\section{Localization of the MCK1 gene}

Restriction digestion analysis showed that $\mathrm{p} M C K 1$ contained a 7-kb yeast genomic DNA insert derived from a Sau3A partial digest inserted into the $B a m H I$ site of pJS62. By standard recombinant DNA techniques, the $M C K 1$ gene was localized through the use of restriction sites unique to the insert and the cloning polylinker to generate intramolecular deletions. In addition, frameshift mutations were generated at unique $N c o I$ and $S p h I$ sites by restriction digest, filling in the ends with Klenow, ligation, and transformation into $E$. coli. These plasmids were assayed for suppressor activity (as above). This analysis identified 
Table 3. Yeast strains for determination of allele-specific suppression of CEN DNA mutations

\begin{tabular}{lc}
\hline Mutation & Yeast strains $^{\mathrm{a}}$ \\
\hline CEN6 (wild type) & YPH281 \\
CDEI $(8-A)^{\mathrm{b}}$ & YPH286 \\
CDEI $^{\mathrm{c}}$ & YPH632 \\
CDEIII $(15-\mathrm{T})^{\mathrm{b}}$ & YPH291 \\
CDEIII $(15-\mathrm{A})^{\mathrm{b}}$ & YPH292 \\
CDEIII $(15-\mathrm{C})^{\mathrm{b}}$ & YPH293 \\
CDEIII $(19 \nabla 20-T)^{\mathrm{b}}$ & YPH298 \\
CDEIII $(19 \nabla 20-G)^{\mathrm{b}}$ & YPH299 \\
CDEIII $(17 \Delta 25)^{\mathrm{b}}$ & YPH300 \\
CDEII $(\Delta 31 \mathrm{bp})^{\mathrm{c}}$ & YPH630 \\
CDEII $(+45 \text { bp })^{\mathrm{c}}$ & YPH631
\end{tabular}

aeast strains have the following genotypes:

YPH281-300: MATa/MAT $\alpha$ ura3-52/ura3-52 lys2-801/lys2-801

ade2-101/ade2-101 trp1 $1 / \operatorname{trp} 1 \Delta 1+C F I I I(D 8 B . d)$

YPH630, 631, 632: MATa/MATa ura3-52/ura3-52 lys2-801/lys2-

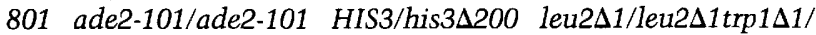
$\operatorname{trp} 1 \Delta 1+C F I I I(D 8 B . d)$.

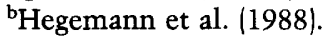

'Panzeri et al. (1985).

a $3.6-\mathrm{kb}$ BamHI-ClaI fragment that had suppressor activity when subcloned into pJS92 to create pJS99. This fragment contained a unique NcoI restriction site that was shown to be located in $M C K 1$-coding sequence by a frameshift mutation that abolished suppressor activity. Low-stringency Southern hybridizations were performed as described (Levin et al. 1987) by using the 3.6-kb BamHI-ClaI genomic fragment as a probe /Feinberg and Vogelstein 1984) to establish that the MCK1 gene was present in single copy in the haploid genome.

\section{Physical and genetic mapping of MCKl}

A 2.0-kb EcoRI-EcoRI DNA fragment derived from the 7.0-kb $M C K 1$-containing DNA fragment was subcloned in both orientations into the EcoRI site of pYCF3 and pYCF4 /Vollrath et al. 1988). Three micrograms of each of the four plasmid DNAs was linearized with BglII before transformation into yeast strain YPH49. Screening of chromosome fragment-containing strains, and subsequent analysis on CHEF gels were performed as described (Gerring et al. 1990). The MCK1 gene was unambiguously placed $80 \mathrm{~kb}$ from the left-arm telomere by hybridization to a cloned DNA segment containing the RPD3 gene (kindly provided by R. Gaber) that hybridized to the $80-\mathrm{kb} M C K 1$-distal chromosome fragment. To obtain a meiotic map position, a diploid strain was constructed by standard procedures, which was heterogygous at multiple loci on XIVL (mck1::HIS3/+, pha2/+, kar1::URA3/+, pet2/+, met2/+1. A total of 112 tetrads were scored for segregation of the markers (all data have been submitted to the Yeast Genetics Stock Center, Berkeley, CA). Significant linkage was observed between mck1 and pha2, met2, and pet2 but not kar1. kar1 was observed to be linked to pet2 but not to any of the other chromosome XIV markers (including mck1), indicating that kar1 is proximal to pet 2 rather than distal as on the current published linkage map. The meiotic distances were calculated from the following data by using the formula of Perkins: mck1-pha2 $18 \mathrm{cM}$ (PD/NPD/T = 76/1/33); mck1-met2, $40 \mathrm{cM}$ (PD/NPD/T = 53/6/51). mck1 was placed proximal to pha2 because (1) pha2 is $8 \mathrm{cM}$ proximal to rpd3 (R. Gaber, pers. comm.), (2) MCK1 is proximal to $r p d 3$ from the Southern blotting data, and (3) mck1::HIS3 is $18 \mathrm{cM}$ from pha2.

\section{Construction of MCK1 disruption allele and DNA sequencing}

A $1.8-\mathrm{kb}$ BamHI DNA fragment containing the HIS3 gene was cloned into the unique NcoI site of $M C K 1$ (pJS99) to generate a disruption allele plasmid (pIS102). A 5.4-kb mck1::HIS3 fragment was excised from pIS102 and transformed into the diploid strain YPH273 to disrupt one copy of the genomic MCK1 locus by homologous recombination (Rothstein 1983). Two independent transformants were selected and shown by Southern blot analysis to have the appropriate disrupted, as well as wild-type, $M C K 1$ genes.

pJS99 contains MCK1 cloned into the BamHI-ClaI sites of the pJS92 polylinker that corresponds to the pBluescript (KS-, Stratagene) polylinker. With the NcoI site as a starting point, a set of unidirectional deletions were made by using the exonuclease III/nuclease S1 method of Henikoff (1987). Additional defined deletions were constructed using restriction enzymes. The DNA sequence encoding the complete open reading frame was determined by the method of Sanger et al. (1977), using double-stranded templates (Hatton and Sakaki 1986). The sequencing of both strands of the open reading frame (Fig. 4) was completed, using synthetic oligonucleotides to cross gaps.

\section{Acknowledgments}

We are grateful to J. Lechner, J. Carbon, L. Neigeborn, and A. Mitchell for sharing their data before publication. We thank $M$. Goebl for homology searches of MCK1 using his data base, and Clarence Chan for pointing out the amino-terminal identity to $T P K 1$. We thank R. Gaber, H. Bussey, and A. Hoyt for strains and plasmids, Carla Connelly for her contributions in mapping $M C K 1$, and F. Spencer, S. Gerring, D. Sears, and A. Burkhoff for comments on the manuscript. The suggestions and help of Bob Sikorski were appreciated. We thank Bertha Robinson and Quentin Burley for their invaluable help in preparing media.

The publication costs of this article were defrayed in part by payment of page charges. This article must therefore be hereby marked "advertisement" in accordance with 18 USC section 1734 solely to indicate this fact.

\section{References}

Axton J.M., V. Dombradi, P.T.W. Cohen, and D.M. Glover. 1990. One of the protein phosphatase 1 isoenzmes in Drosophila is essential for mitosis. Cell 63: 33-46.

Baker, R.E., M. Fitzgerald-Hayes, and T.C. O’Brien. 1989. Purification of the yeast centromere binding protein $\mathrm{CP} 1$ and a mutational analysis of its binding site. $J$. Biol. Chem. 264: 0843-10850.

Bloom, K. and J. Carbon. 1982. Yeast centromere DNA is in a unique and highly ordered structure in chromosomes and small circular minichromosomes. Cell 29: 305-317.

Boeke, J., J. Truehart, G. Natsoulis, and G. Fink. 1987. 5-Fluoroorotic acid as a selective agent in yeast molecular genetics. Methods Enzymol. 154: 164-175.

Botstein, D. and R. Maurer. 1982. Genetic approaches to the analysis of microbial development. Annu. Rev. Genet. 16: 61-83.

Bourouis, M., P. Moore, L. Ruel, Y. Grau, P. Heitzler, and P. 
Simpson. 1990. An early embryonic product of the gene shaggy encodes a serine/threonine protein kinase related to the CDC28/cdc2 + subfamily. EMBO J. 9: 2877-2884.

Bram, R.J. and R.D. Kornberg. 1987. Isolation of a Saccharomyces cerevisiae centromere DNA- binding protein, its human homolog, and its possible role as a transcription factor. Mol. Cell. Biol. 7: 403-409.

Cai, M. and R.W. Davis. 1990. Yeast centromere binding protein CBF1, of the helix-loop-helix protein family, is required for chromosome stability and methionine prototrophy. Cell 61: 437-446.

Clarke, L. and J. Carbon. 1980. Isolation of a yeast centromere and construction of functional small circular chromosomes. Nature 287: 504-509.

Dailey, D., G.L. Schieven, M.Y. Lim, H. Marquardt, T. Gilmore, J. Thorner, and G.S. Martin. 1990. Novel yeast protein kinase (YPKl gene product) is a 40-kilodalton phosphotyrosyl protein associated with protein-tyrosine kinase activity. Mol. Cell. Biol. 10: 6244-6256.

Davis, F.M., T.Y. Tsao, S.K. Fowler, and P.N. Rao. 1983. Monoclonal antibodies to mitotic cells. Proc. Natl. Acad. Sci. 80: $2926-2930$.

Davis, R.W., D. Botstein, and J. Roth. 1980. Advanced bacterial genetics. Cold Spring Harbor Laboratory, Cold Spring Harbor, New York.

Dotto, G.P. and N.D. Zinder. 1984. Increased intracellular concentration of an initiator protein markedly reduces sequence required for initiation of DNA synthesis. Proc. Natl. Acad. Sci. 81: 1336-1340.

Feinberg, A.P. and B. Vogelstein. 1984. A technique for radiolabeling DNA restriction endonuclease fragments to high specific activity. Anal. Biochem. 137: 266-267.

Gerring, S., C. Connelly, and P. Hieter. 1990 Positional mapping of cloned genes by chromosome blotting and chromosome fragmentation. Methods Enzymol. 194: 57-77.

Hadwiger J.A., C. Wittenberg, M.D. Mendenhall, and S.I. Reed. 1989. The Saccharomyces cerevisiae CKS1 gene, a homolog of the Schizosaccharomyces pombe suc1 + gene, encodes a subunit of the CDC28 protein kinase complex. Mol. Cell. Biol. 9: 2034-2041.

Hanahan, D. 1983. Studies on transformation of Escherichia coli with plasmids. J. Mol. Biol. 166: 557-580.

Hanks, S.K., A.M. Quinn, and T. Hunter. 1988. The protein kinase family: Conserved features and deduced phylogeny of the catalytic domains. Science 241: 42-52.

Hartwell, L. and T. Weinert. 1989. Checkpoints: Controls that ensure the order of cell cycle events. Science 246: 614-621.

Hattori, M. and Y. Sakaki. 1986. Dideoxy sequencing method using denatured plasmid templates. Anal. Biochem. 152: 232-238.

Hayles J., D. Beach, B. Durkacz, and P. Nurse. 1986. The fission yeast cell cycle control gene cdc2: Isolation of a sequence suc1 that suppresses $c d c 2$ mutant function. Mol. Gen. Genet. 202: 291-293.

Hegemann J., R. Pridmore, R. Schneider, and P. Phillippsen. 1986. Mutations in the right boundary of Saccharomyces cerevisiae centromere VI lead to nonfunctional or partially functional centromeres. Mol. Gen. Genet. 205: 305-311.

Hegemann J., J.H. Shero, G. Cotteral, P. Philippsen, and P. Hieter. 1988. Mutational analysis of centromere DNA from chromosome VI of Saccaromyces cerevisiae. Mol. Cell. Biol. 8: 2523-2535.

Henikoff, S. 1987. Unidirectional digestion with exonuclease III in DNA sequence analysis. Methods Enzymol. 155: 156165.

Hieter, P., C. Mann, M. Snyder, and R. Davis. 1985. Mitotic stability of yeast chromosomes: A colony color assay that measures nondisjunction and chromosome loss. Cell 40: 381-392.

Holmes, D.S. and M. Quigley. 1981. A rapid boiling method for the preparation of bacterial plasmids. Anal. Biochem. 114: 193-197.

Hoyt, M.A., T. Stearns, and D. Botstein. 1990. Chromosome instability mutants of Saccharomyces cerevisiae that are defective in all microtubule mediated processes. Mol. Cell. Biol. 10: 223-234

Ito, H., Y. Fukuda, K. Murata, A. Kimera. 1983. Transformation of intact cells treated with alkali cations. I. Bacteriol. 153: $163-168$.

Jiang, W. and P. Philippsen. 1989. Purification of a protein binding to the CDEI subregion of Saccharomyces cerevisiae centromere DNA. Mol. Cell. Biol. 9: 5585-5593.

Lea, D. and C. Coulson. 1949. The distribution of numbers of mutants in bacterial populations. J. Genet. 49: 264-285.

Lechner, J. and J. Carbon. 1991. A 240-kD multisubunit protein complex (CPF3) is a major component of the budding yeast centromere. Cell (in press).

Levin, D.E., C.I. Hammond, R.O Ralston, and J.M. Bishop. 1987. Two yeast genes that encode unusual protein kinases. Proc. Natl. Acad. Sci. 84: 6035-6039.

Lipman, D.J. and W.R. Pearson. 1985. Rapid and sensitive protein similarity searches. Science 227: 1435-1441.

Lohka, M.K., I.L. Kyes, and J.L. Maller. 1987. Metaphase protein phosphoryiation in Xenopus laevis eggs. Mol. Cell. Biol. 7: 760-768

Magasanik, B. 1988. Reversible phosphorylation of an enhancer binding protein regulates the transcription of bacterial nitrogen utilization genes. Trends Biochem. Sci. 13: 475-479.

Maniatis, T., E.F. Fritsch, and J. Sambrook. 1982. Molecular cloning: A laboratory manual. Cold Spring Harbor Laboratory, Cold Spring Harbor, New York.

Newlon, C. 1988. Yeast chromosome replication and segregation. Microbiol. Rev. 52: 568-601.

$\mathrm{Ng}, \mathrm{R}$. and J. Carbon. 1987. Mutational and in vitro proteinbinding studies on centromere DNA from Saccharomyces cerevisiae. Mol. Cell. Biol. 7: 4522-4534.

Nurse, P. 1990. Universal control mechanism regulating onset of M-phase. Nature 344: 503-508.

Panzeri, L., L. Landonio, A. Stotz, and P. Philippsen. 1985. Role of conserved sequence elements in yeast centromere DNA. $E M B O$ J. 4: 1867-1874.

Peterson, J. and H. Ris. 1976. Electron microscope study of the spindle and the chromosome movement in the yeast $S$. cerevisiae. I. Cell Sci. 22: 219-242.

Prywes, R., A. Dutta, J.A. Cromlish, and R.G. Roeder. 1988. Phosphorylation of serum response factor, a factor that binds to the serum response element of the c-FOS enhancer. Proc. Natl. Acad. Sci. 85: 7206-7210.

Raychauduri, P., S. Bagchi, S. and J. Nevins. 1989. DNA-binding activity of the adenovirus-induced E4F transcription factor is regulated by phosphorylation. Genes \& Dev. 3: 620-627.

Rothstein, R.J. 1983. One-step gene disruption in yeast. Methods Enzymol. 101: 202-211.

Sanger, F., S. Nicklen, and A. Coulson. 1977. DNA sequencing with chain terminating inhibitors. Proc. Natl. Acad. Sci. 74: 5463-5467.

Sherman, F., G.R. Fink, and C.W. Lawrence. 1978. Methods in yeast genetics. Cold Spring Harbor Laboratory, Cold Spring Harbor, New York.

Shero, J.H., M. Koval, F. Spencer, R. Palmer, P. Hieter, and D. Koshland. 1991. Analysis of chromosome segregation in Saccharomyces cerevisiae. Methods Enzymol. 194: 749-774. 
Shortle, D.R., R.F. Margolskee, and D. Nathans. 1979. Mutational analysis of simian virus 40 replication: Pseudorevertants of mutants with a defective replication origin. Proc. Natl. Acad. Sci. 76: 6128-6131.

Sikorski, R.S. and P. Hieter. 1989. A system of shuttle vectors and yeast host strains designed for efficient manipulation of DNA in Saccharomyces cerevisiae. Genetics. 122: 19-27.

Spencer, F., S.L. Gerring, C. Connelly, and P. Hieter. 1990. Mitotic chromosome transmission fidelity mutants in Saccharomyces cerevisiae. Genetics 124: 237-249.

Stearns, T., M.A. Hoyt, and D. Botstein. 1990. Yeast mutants sensitive to anti-microtubule drugs define three genes that regulate microtubule function. Genetics 124: 251-262.

Thomas J., N. Neff, and D. Botstein. 1985. Isolation and characterization of mutations in the $\beta$-tubulin gene of Saccharomyces cerevisiae. Genetics. 112: 715-734.

Toda, T., S. Cameron, P. Sass, M. Zoller, and M. Wigler. 1987. Three different genes in $S$. cerevisiae encode the catalytic subunits of the cAMP-dependent protein kinase. Cell 50: 277-287.

Vandre, D.D., F.M. Davis, P.N. Rao, and G.G. Borisy. 1984. Phosphoproteins are components of mitotic microtubule organizing centers. Proc. Natl. Acad. Sci. 81: 4439-4443.

Vollrath, D., R. Davis, C. Connelly, P. Hieter. 1988. Physical mapping of large DNA by chromosome fragmentation. Proc. Natl. Acad. Sci. 85: 6027-6031.

Westwood J.T., R.B. Church, and E.B. Wagenaar. 1985. Changes in protein phosphorylation during the cell cycle of Chinese hamster ovary cells. J. Biol. Chem. 260: 10308-10313.

Woodgett, J.R. 1990. Molecular cloning and expression of glycogen synthase kinase-3/factor A. EMBO. I. 9: 2431-2438.

Yamamoto, K.K., G.A. Gonzalez, W.H. Biggs III, and M.R. Montminy. 1988. Phosphorylation- induced binding and transcriptional efficacy of nuclear factor CREB. Nature 334: 494-498. 


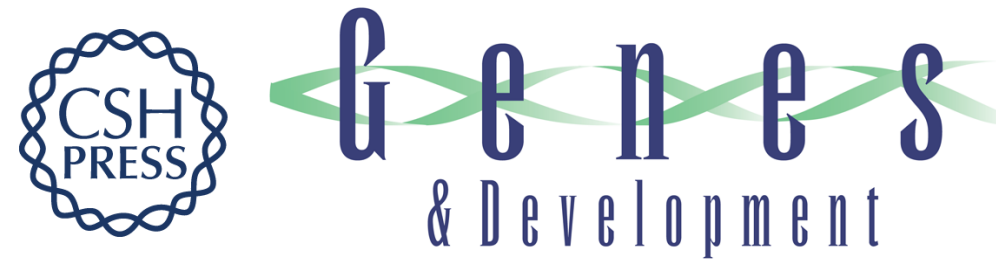

\section{A suppressor of a centromere DNA mutation encodes a putative protein kinase (MCK1).}

$\mathrm{J} \mathrm{H}$ Shero and $\mathrm{P}$ Hieter

Genes Dev. 1991, 5:

Access the most recent version at doi:10.1101/gad.5.4.549

References This article cites 54 articles, 27 of which can be accessed free at:

http://genesdev.cshlp.org/content/5/4/549.full.html\#ref-list-1

License

Email Alerting

Service

Receive free email alerts when new articles cite this article - sign up in the box at the top right corner of the article or click here.

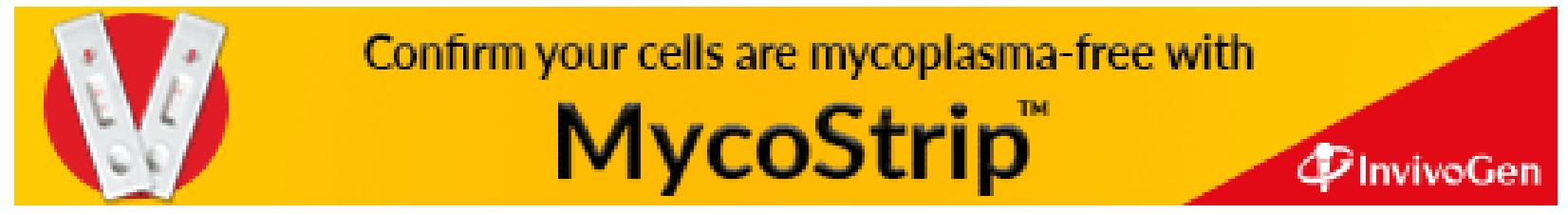

